Ann. Zootech., I962, 11 (2), I5I-I56.

\title{
NOUVEAU CAS DE POLYDACTYLIE HÉRÉDITAIRE CHEZ LES BOVINS
}

\author{
J. J. LAUVERGNE \\ Station de Génétique animale, \\ Centre national de Recherches zootechniques, Jouy en Josas, (Seine-et-Oise).
}

\section{SOMIMAIRE}

Une forme de polydactylie héréditaire a été observée dans la descendance d'un Taureau Normand normal. La tare se manifeste par l'adjonction d'un doigt supplémentaire préaxial ; elle atteint les pattes antérieures et accessoirement les pattes postérieures; on trouve des variations dans son degré d'expression.

Cette tare, qui atteint les deux sexes, est observée sur 2 p. Ioo des descendants femelles, l'hypothèse génétique la plus vraisemblable est qu'il s'agit d'un facteur dominant incomplètement pénétrant.

\section{INTRODUCTION}

La polydactylie, définie comme étant la présence d'un nombre de doigts supérieur au nombre de doigts de l'espèce, semble être courante dans le règne animal.

BROCHART (I945) a fait une revue bibliographique des anomalies du segment distal chez l'homme et les animaux domestiques. La polydactylie a été signalée aussi bien chez l'Homme que chez le Cheval, le Bœuf, le Mouton, la Chèvre, le Porc, le Chien, le Chat, le Cobaye et la Poule. Elle se rencontre également chez la Souris (GRÜNEBERG, I952), le Pigeon (WIESNER, I960), chez divers batraciens (RosTAND, I95I) et certainement dans beaucoup d'autres espèces animales.

Nous avons vu apparaître la polydactylie dans la descendance du taureau normand QUIDAm, en cours de testage au Centre d'Insémination artificielle de Charmor (Yonne). Nous avons examiné au 3I mars I96I, I22 femelles parmi lesquelles 2 étaient polydactyles ; un mâle polydactyle a été également observé.

\section{ÉTUDE, MORPHOLOGIQUE}

\section{I $^{\circ}$ Le segment distal de Bos Taurus}

L'examen du squelette osseux des membres de Bos Taurus nous révèle selon Chauveau et ARIoING (I903) un carpe formé de 6 os et un tarse formé de 5 os prolongés l'un et l'autre par l'os canon issu de la soudure des métacarpiens ou métatar- 
siens III et IV ; le métacarpien I est absent, le II presque toujours absent, le V très réduit ; le métatarsien I est toujours absent, les II et $\mathrm{V}$ sont très réduits et soudés à l'os canon. Les doigts III et IV à l'avant comme à l'arrière sont fonctionnels et comportent trois phalanges. On trouve, en outre, des os dits sésamoïdes, 4 au niveau de la première phalange formant la base des 2 ergots et 2 au niveau de la deuxième phalange (tableau I).

TABLEAU I

Os formant les cxtrémités distales des membres de Bos Taurus

\begin{tabular}{|c|c|c|}
\hline & Main & Pied \\
\hline Carpe ou tarse & 6 os & 5 os \\
\hline Métacarpe ou métatarse & $\begin{array}{l}\text { I absent } \\
\text { II presque toujours absent } \\
\text { III et IV os canon } \\
V \text { trìs réduit }\end{array}$ & $\begin{array}{l}\text { I absent } \\
\text { II soudé au canon } \\
\text { III et IV os canon } \\
\text { V soudé au canon }\end{array}$ \\
\hline Doigts & $\begin{array}{l}\text { II I complet } \\
\text { IV complet } \\
\text { \& grands sésamoïdes } \\
2 \text { petits sésamoïdes }\end{array}$ & $\begin{array}{l}\text { III complet } \\
\text { IV complet } \\
\text { \& grands sésamoïdes } \\
2 \text { petits sésamoïdes }\end{array}$ \\
\hline
\end{tabular}

$2^{\circ}$ Description des anormaux descendants de QUIDAM

Les renseignements concernant les deux sujets anormaux femelle sont rassemblés dans le tableau 2 .

TABLEAU 2

Référence des génisses anormales dans la descendance de Quidam

\begin{tabular}{|c|c|c|c|c|}
\hline $\begin{array}{l}\text { No de référence } \\
\text { CIA Charmoy }\end{array}$ & Nom & Date de naissance & Naisseur & Anomalie \\
\hline $606019: 315$ & Fleurette & juillet-aoùt 1960 & $\begin{array}{l}\text { Bertiox Pierre, ferme de } \\
\text { Bellevue, Viglain (Loi- } \\
\text { ret) }\end{array}$ & 3 onglons aux 4 pattes \\
\hline 606035315 & Coquette & aout-septembre 1960 & $\begin{array}{l}\text { Chisss Jean, Villechétive } \\
\text { (Youne) }\end{array}$ & $\begin{array}{l}3 \text { onglons aux } 2 \text { pattes } \\
\text { antérieures }\end{array}$ \\
\hline
\end{tabular}

Leur examen à l'âge de II mois n'a pas révélé d'autres anomalies que la polydactylie qui a persisté depuis. Les radiographies et photos ont été faites lorsque les animaux étaient âgés de I 8 mois environ.

- or9 présente 3 doigts aux quatre pattes (fig. I et 2) :

aux pattes antérieures le doigt supplémentaire est intérieur; il a 3 phalanges et ne repose pas sur le sol; à la radiographie on remarque la présence d'un métacarpien très mince (fig. I et 2 ) ; à gauche la patte conserve 2 ergots alors qu'à droite il n'y en a plus qu'un ;

aux pattes postérieures, le doigt supplémentaire est également intérieur ; il est moins développé qu'aux pattes antérieures ; la radiographie révèle la présence 

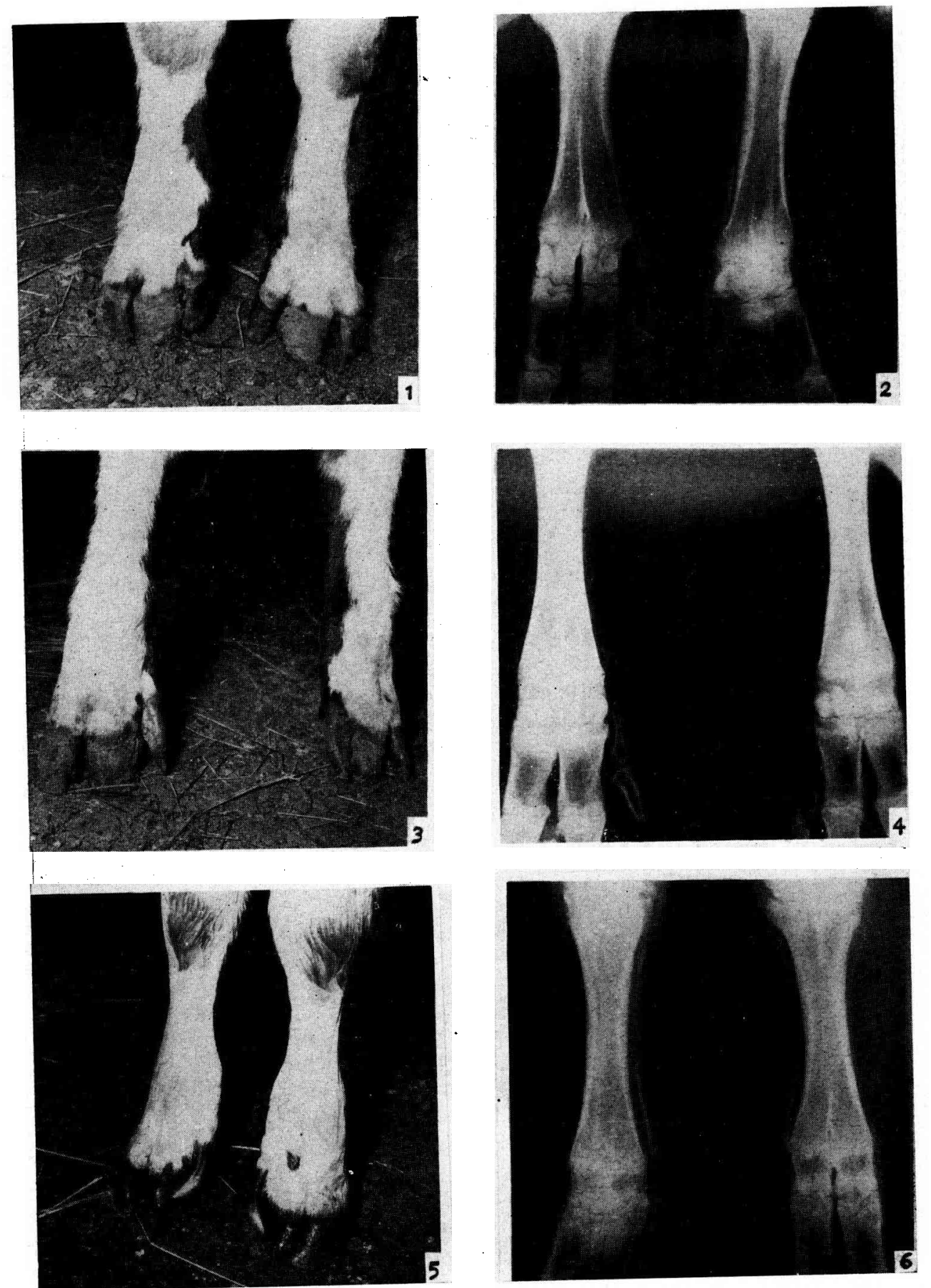

Fic. I et 2.- Pattes antérieures de oro, rue te face Fig. 3 et 4. - Pattes posterientes de org, rue de fice Fig. 5 et 6. - Pattes antérieures de 035 , rue de tace 
beaucoup plus fruste du métatarsien intérieur, de 2 phalanges à droite et de 3 phalanges à gauche (fig. 3 et 4 ); il y a un seul ergot à chaque patte.

- 035 présente 3 doigts aux pattes antérieures seulement, il s'agit là encore d'un doigt intérieur non fonctionnel; les images radiologiques sont très semblables à celles des pattes avant de org (métacarpien interne filiforme, 3 phalanges) ; on note, en outre à gauche, la présence du métacarpien rudimentaire IV (fig. 5 et 6). Les deux pattes ne possèdent qu'un seul ergot.

Chez le veau mâle polydacty-le, l'anomalie se limitait aux pattes antérieures qui présentaient trois onglons.

\section{$3^{\circ}$ Discussion}

Brochart (I945) remarque que la variété des manifestations de l'anomalie avait conduit les anciens auteurs anatomistes et tératologistes à distinguer plusieurs types de polydactylie : il y aurait d'abord une polydactylie atavique, qui engloberait les cas de réapparition de doigts perdus au cours de l'évolution, il y aurait en outre une polydactylie par dédoublement des doigts, puis par dédoublement de la main ou du pied.

Toute étude héréditaire de la polydactylie conduit à la conclusion que cette dernière peut se manifester sous des formes très différentes. Il n'est pas logique de créer une terminologie de classification par avance arbitraire. Ainsi, chez les bovins, RoBER'TS (I92I) trouvait dans une même lignée d'Holstein Friesian aux U. S. A. des cas dits de tridactylie et un autre de diplopodie (dédoublement du pied).

Nous admettons, pour notre compte, que cela représente un setıl et même phénomène, puisqu'aussi bien nous avons décrit des variations dans l'expression phénotypique de cette tare.

Il nous semble donc logique, autant que prudent, de définir ainsi la polydactylie que nous avons observée :

Polydactylie préaxiale par réapparition d'un doigt à l'emplacement du doigt disparu no II, qui se manifeste toujours anx pattes antérieures, plus rarement et moins intensément aux pattes postérieures; les membres atteints présentent des variations dans le nombre des ergots.

\section{ÉTUDE: GÉNÉTIQUE}

Dans la descendance de QCiDAM, taureau normal croisé à des vaches Normandes également normales, on a trouvé des anormaux des deux sexes. La fréquence de ces apparitions n'a pu être établie que chez les femelles, elle est de $2:$ I 22 soit I,8 p. Ioo.

ROBERTS (I92I) déjà cité observe des cas de polydactylie dans trois générations successives et il constate l'apparition d'anormaux dans des croisements d'anormaux avec normaux, d'où il conclut à la présence d'un seul facteur dominant.

MORRIL. (I945) dans la race Heretord aux U.S. A. ne trouve l'anomalie que chez des mâles, fils ou petits-fils par leur mère d'une même vache; il avance 1'hypothèse d'un facteur récessif lié au sexe. 
Dans les deux cas le nombre total d'animaux observés est faible (moins d'une dizaine).

Ces deux auteurs ne mettent pas en doute la monofactorialité de l'anomalie; considérant la très grande récurrence de la tare qui a été signalée de tous temps dans presque toutes les races (les musées tératologiques contiennent de nombreuses pièces anatomiques de cette anomalie), et en raisonnant par analogie avec les autres espèces où 1'analyse génétique a été poussée plus loin, il semble logique d'adopter cette hypothèse, au moins momentanément.

Quant au mode d'action du gène nous ne pouvons, pour expliquer le cas relaté, ni retenir 1'hypothèse de dominance (ROBERTS, I92I) ni l'hypothèse de récessivité liée au sexe (MORRILI, I954).

Il nous paraît donc logique d'approfondir la bibliographie de la question, en nous référant à une espèce beaucoup mieux étudiée. GRÜNEBERG (I952) nous apprend que chez la souris existent au moins 4 cas de polydactylie préaxiale et 2 cas de polydactylie postaxiale simple, sans compter les anomalies bien caractérisées qui s'accompagnent accessoirement de polydactylie, telles que les tares myolencephalic blebs ou fidget.

Étudiées dans des lignées différentes les polydactylies préaxiales simples c'est-à-dire non accompagnées d'autres anomalies - manifestent une variabilité d'expression assez grande et leurs disjonctions ne peuvent se ramener à des rapports mendéliens simples, au moins à première vue. Cependant HoLT (I945), dans le cas qu'il étudie, montre qu'il s'agit d'un seul facteur mendélien incomplètement récessif.

Cette notion de récessivité ou de dominance incomplète avait d'ailleurs été dégagée depuis longtemps par TIMOFEEFF-RESSOVSKy (I925), cité par BROCHART (I945), qui avançait les termes de "pénétrance " comme mesure du pourcentage d'anormaux parmi les porteurs d'une certaine combinaison génétique, et d'“ expressivité " comme traduction des différences pouvant se manifester dans l'action du gène.

Dans des anomalies où l'expressivité est très variable on conçoit très bien qu'à la limite certains tarés ne soient pas décelables parce que trop faiblement atteints ; ils sont dits normal overlap (CLARK, I934, cité par KoBozIEFF, I939).

Ces notions de pénétrance incomplète et d'expressivité variable sont très utiles, mais elles traduisent notre ignorance des circonstances exactes capables de modifier l'expression du gène majeur, responsable de l'anomalie.

Cette situation a pu cependant être précisée dans le cas du polydactylisme de HoLT par la mise en évidence par Fisher (I950) d'un gène susceptible de réduire ro fois la manifestation de l'anomalie.

La palydactylie se classe d'ailleurs parmi les anomalies dont on peut obtenir une phénocopie. Ainsi Evans et al. (I95I), en remplaçant à un certain stade de la gestation l'acide folique par un antimétabolite dans la ration de la rate gravide font apparaitre dans la descendance toutes sortes d'anomalies, dont la polydactylie.

En raisonnant par analogie avec des espèces mieux étudiées on peut avancer à côté de l'hypothèse de récessivité complète, celles de dominance ou de récessivité à pénétrance incomplète.

L'hypothèse de récessivité impliquerait qu'une partie du cheptel femelle soit porteuse du gène responsable de la polydactylie alors que 1'hypothèse de dominance ne nécessiterait pas cette supposition. Remarquons d'ailleurs que les deux cas de ROBERTs (I92I) et de MORRILL (I945) pourraient s'expliquer par 
une dominance à pénétrance incomplète. Encore faudrait-il démontrer que tous les cas de polydactylie bovine rencontrés proviendraient de mutations au même locus. Disons qu'avec nos données la dominance à pénétrance incomplète est plus vraisemblable que la récessivité à pénétrance incomplète. La pénétrance du zygote chez les hétérozygotes serait alors de 4 p. Ioo.

Reçu en mars 1962

\section{SUMMARY}

\section{A NEW CASE OF HEREDITARY POLYDACTYLISM IN CATTLE}

A form of hereditary polydactylism has been observed in the progeny of a normal bull from the "Normande " breed. The defect appears as an additional preaxial digit. It is found on the forefeet and as accessories on the hind feet. Variations have been noted in the degree of its manifestation.

This defect which may affect both sexes has been observed in 2 p. 100 of the female descendants, the most likely genetical hypothesis being that it ist he result of an dominant factor with incomplete penetrance.

\section{RÉFÉRENCES BIBLIOGRAPHIQUES}

Brochart M., 1945. Les anomalies congénitales et héréditaires du squelette des membres des Animaux domestiques et de l'Homme (segment acromélique ou distal), Thèse Vétérinaire, R. Foulon, Paris.

Chauveau A., Arlong S., igo3. Traité d'anatomie comparée des animaux domestiques, Baillère et Fils, Paris.

Clark F. H., 1934. Genetics, 19, 365-393.

Evans H. M., Nelson M. M., Asling C. W., 195I. Multiple congenital abnormalities resulting from acute folic acid deficiency during gestation, Science, 114, 479 .

Fisher R. A., 1950. Polydactylism in mice, Nature, 165, 407.

Grüneberg H., I952. The Genetics of the Mouse, Martinus Nijhoff, La Haye.

Holt. Cité par GrüneberG (1952).

Kobozieff N., Pomriaskinsky-Kobozieff N. A, i939. Sur la constitution généotypiques de la brachyourie accompagnée de coudures chez la Souris. Bull Biol. 78, 330-335.

Morill, E. L., i945. A new sex-linked defect in cattle, J.Hered., 36, 8I-82.

Roberts E. J., I9zi. Polydactylism in cattle. J. Hered., 12, 84-86.

Rostand J., I95I. Sur la polydactylie des batraciens anoures. Bull. Biol., 85, I I $3^{-\mathrm{I}} 3^{6}$.

Trmofeeff-Ressovsky N. W., I925. Studies on the phenotypic manifestation of hereditary factors. The genovariation radius incompletus of Drosophila funebris. Zhurn. Eskp. Biol.

Wiesner E., 1960. Die Erbschäden der landairtschaftlichen Nutztiere, Gustav FISHer, Jena. 